Results The analysis showed that there is a moderate and negative correlation between WAI and fatigue $(r=-0.49)$, showing an association between lower values of WAI and higher values of fatigue. The working week bigger than $36 \mathrm{~h}$ showed association with the WAI $(\mathrm{p}=0.009)$. Working days less than $36 \mathrm{~h}$ reduce, on average, 4-point score of WAI. Women presented higher tendency for fatigue $(55.7 \%$ vs $25.0 \%$ for men) or lower average to WAI (43.1\% vs $45.8 \%$ for men). There was no significant difference for the WAI $(p=0.246)$ and fatigue $(0.752)$ among workers with or without work restriction.

Conclusion There is a significant correlation between work ability and fatigue of nursing personnel, however it was noted that there is no significant differences of these indexes, considering both workers with or without work restriction.

\section{OCCUPATIONAL FACTORS ASSOCIATED WITH LATENT TUBERCULOSIS INFECTION AND CONVERSION IN HEALTH CARE WORKERS IN A HIGH TUBERCULOSIS/HIV PREVALENCE SETTING}

${ }^{1} \mathrm{R}$ I Ehrlich, ${ }^{2}$ Adams, ${ }^{2}$ Van Zyl Smit, ${ }^{3}$ Said-Hartley, ${ }^{2}$ Dawson, ${ }^{4}$ Dheda. ${ }^{1}$ Division of Occupational Medicine, Cape Town, South Africa; ${ }^{2}$ UCT Lung Institute, Cape Town, South Africa; ${ }^{3}$ Deparment of Radiology, University of Cape Town, Cape Town, South Africa; ${ }^{4}$ Division of Pulmonology, Department of Medicine, University of Cape Town, Cape Town, South Africa

\subsection{6/oemed-2013-101717.169}

Objective South African health care workers are at growing risk of tuberculosis (TB). This study sought occupational risk factors for latent TB infection (LTBI).

Methods A sample of public sector facility staff in Cape Town completed a questionnaire and underwent 3 tests for LTBI: (1) tuberculin skin test (TST) (skin induration $>10 \mathrm{~mm}$ ) (2) QuantiFERON-TB Gold In-Tube (QFT-GIT) and (3) TSPOT. TB test. These were repeated one year later and annual rate of test conversion calculated. Occupational factors associated with baseline LTBI and conversion were sought, adjusting for age and gender and stratified by primary care vs TB hospitals.

Results 505 staff participated from 7 facilities. LTBI prevalence was high: TST 84\%; QFT-GIT 65\%; and TSPOT. TB $60 \%$. Predictors of positive TST in primary care were employment duration $>20$ years $[\mathrm{OR}=4.17(95 \%$ CI $1.12-15.62)$; hospital staff with training on self-protection from TB infection were less likely to test positive $[\mathrm{OR}=0.38(0.16-0.91)]$. Predictors of a positive QFT-GIT test in primary care were involvement in sputum collection [OR $=3.25(1.28-8.09)$ ] and employment $>20$ years $[\mathrm{OR}=2.42(1.09-5.38)]$, while again there was a protective training effect in hospital staff $[\mathrm{OR}=0.41(0.22-0.77)]$. Predictors of a positive TSPOT. TB in primary care were providing home-based care to $\mathrm{TB}$ patients $[\mathrm{OR}=4.14(1.60-10.70)]$, and, paradoxically, working at a facility which advocated cough etiquette [OR = $2.06(1.04-4.10)]$ or provided surgical masks to coughing patients $[\mathrm{OR}=3.65(1.16-11.51)$. The conversion rates were: TST 38\% (95\% CI 24-55) and QFT-GIT and TSPOT. TB both 22\% (15-30). There were no consistent occupational predictors of conversion.

Conclusion LTBI prevalence and conversion are very high in this population, suggesting occupational risk. Occupational factors included duration and intensity of exposure (primary care, sputum collection, home visits), suggesting targets for infection control. However, more research is needed on occupational risk.

\section{0}

ASSOCIATION BETWEEN EXPOSURE TO MRI-RELATED MAGNETIC STRAY FIELDS AND SYMPTOMS REPORTED BY WORKERS IN THE PUBLIC HEALTH AND RESEARCH SECTOR

K Schaap, Mason, Christopher - De Vries, Kromhout. Utrecht University, Utrecht, The Netherlands

10.1136/oemed-2013-101717.170

Objectives This study aims to assess which acute symptoms are prevalent among health care and research staff working with MRI scanners, and whether these are related to their exposure to static magnetic stray fields.

Methods Fourteen health care and research MRI facilities were surveyed. Full-shift measurements of exposure to static and timevarying magnetic fields (SMF and TVMF) among staff were collected using personal dosimeters. Participants filled out one to two forms per shift, on which they reported their activities and symptoms they had experienced during (part of) their working day. Fourteen target symptoms were included which had been reported in literature in association with exposure to MRIrelated SMF and TVMF . A subgroup of five 'core' symptoms was defined based on stronger (statistical) evidence for their association with SMF and TVMF exposure. Six additional unrelated symptoms were included to control for over-reporting of symptoms in general.

Results In total, 1,056 forms were completed by 334 participants. Nine out of 14 target symptoms were reported more frequently among staff exposed to SMFs, compared to unexposed staff. The proportion of forms on which at least one target symptom was reported increased with increasing scanner field strength. This trend was even stronger when focusing on the five 'core' symptoms. Strongest associations were seen for nausea, vertigo, metallic taste, and feeling of instability. No association was seen for the subgroup of unrelated symptoms.

Conclusions An increased number of symptoms was reported by staff working in the static magnetic stray field of an MRI-scanner. A clear trend of increased symptom reporting with increasing scanner field strength underlines the potential that a causal relation exists between exposure to MRI stray fields and specific symptoms.

\section{HEALTH RISK WITH WORKING INFECTIOUS WASTE TRANSPORTATION FROM HOSPITAL BY PRIVATE TRANSPORT SECTOR OF THAILAND}

${ }^{1}$ Hansakul, ${ }^{2}$ Pitaksanurat. ' Nakhon Naiyok, Thailand; ${ }^{2}$ Khonkaen University, Khonkaen, Thailand

\subsection{6/oemed-2013-101717.171}

This study aims to identify problems and the health risk with worker who work with the infectious waste transportation (IWT) from hospital by private transport sector (PTS) in Thailand. The instrument test content validity analysis $(\mathrm{IOC}=0.79$ ), reliability analysis (Cobarch's alpha $=0.82$ ). The percentage, mean, standard division, and logistic regression were employed to describe the data. The results are as follow:

Total of 127 workers from 13 PTSs in Thailand $86.6 \%$ of workers were male, mean age of 31 years old, most of them were married $(71.7 \%)$ and the mean of working were 5.03 years. The main work were collector of $57.5 \%$, drivers of $26 \%$, both driver and collector of $16.5 \%$. The mean driving time of 8 hrs a day (min: $\max =2: 24$ hrs). $61.1 \%$ of the driver have had 
rotated every $4 \mathrm{hr}$. Only 38.6\% was trained (control of precaution and protection) by the Ministry of Public Health. In one year later, The worker had an accident or injury (punch with needle or other sharp) from IW during working 42.5\%, 37.8\% have had contaminated or touch with the infectious fluid, $18.9 \%$ had traffic accident (car turnover, clash, offside cone) and 8.3\% had the IW or leachates flood pour out from vehicle or container. The factors associated with risk of the health of the IW worker taken into account of affect of other factors, it were found that prevention and precaution training, job position, age and yearly health check were statistical significant with the health risk, it were found that prevention and precaution training $(\mathrm{OR}=4.61)$ job position $(\mathrm{OR}=3.68)$, Age $(\mathrm{OR}=2.97)$ and yearly health check $(\mathrm{OR}=0.96)$ were statistical significant with the health risk. The problems of IW transportation from hospital by PTS were health risk. The infectious waste management (IWM) which organised by PTS need to develop for better system and quality.

\section{HEALTHCARE WORKERS AND OCCUPATIONAL EXPOSURE TO ANTINEOPLASTIC DRUGS IN ONCOLOGY WARDS}

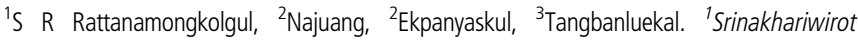
University, Nakhon Nayok, Thailand;' ${ }^{2}$ Faculty of Medicine, Srinakharinwirot Univesity, Nakhon Nayok, Thailand; ${ }^{3}$ Faculty of Medicine. Siriraj Hospital, Mahidol University, Bangkok, Thailand

\subsection{6/oemed-2013-101717.172}

Objective To quantify levels of Ifosfamide (IF) in urine among healthcare workers in a hospital setting.

Methods This cross-sectional study identified 70 subjects who handled IF in oncology wards or antineoplastic drug preparing units at a medical school hospital. Their urine samples were collected at 1-2 hours prior to the end of each work shift, the levels of IF was determinate by gas chromatography mass spectrometer (GC/MS). Information on demographic information, work practice, work position, working station, medication timing and dosages including the time of urine collections was collected using self-administered questionnaires and personal interviews.

Results IF levels were detected in urine of 20 personnel $(28.6 \%$ of total) with geometric mean of $3.6 \mathrm{ng} / \mathrm{mL}$, arithmetic mean $43.6 \mathrm{ng} / \mathrm{mL}$, min-max of $16-526 \mathrm{ng} / \mathrm{mL}$. Among IF-positive subjects, $53.8 \%$ of workers with were in the age group of 41-60 years. Levels of IF were found in all subjects who were drug-preparing workers were found positive tests for IF. The levels of urine IF (geometric mean) were found to be of $526 \mathrm{ng} / \mathrm{mL}$ among the pharmacist assistants, $3.1 \mathrm{ng} / \mathrm{mL}$ for cleaning worker, $2.9 \mathrm{ng} / \mathrm{mL}$ for nurses, and $86.8 \mathrm{ng} / \mathrm{mL}$ for pharmacists.

Conclusions All groups of healthcare workers in the processes of anti-neoplastic medication especially among pharmacist assistants and cleaning workers. Although in a high standard protection against the oncological chemicals, health personnel are at risk of contaminations especially among professional assistants and general workers.

\section{POSSIBLE HIV EXPOSURE: LABORATROY WORKERS KNOWLEDGE ATTITUDES AND BEHAVIOUR}

K S Wilson, Zungu, Nattey. NIOH, Johannesburg, South Africa

10.1136/oemed-2013-101717.173
A company wide KAP survey was conducted around HIV, but in this workplace, exposure to HIV through work exposures was also possible. In a high prevalence HIV country workers perceptions and behaviour around needlesticks play a role in the occupational health and safety

Methods This survey was nested within the larger KAP survey which was distributed to all employees of the company with their pay slips. The questions covered knowledge of correct procedures to follow, likely hood to take prophylaxis and complete the course.

Results 1497 employees participated in the KAP survey which covered all regions of South Africa and represented all occupations within the company. One third of participants were only partly aware or not al all aware of the correct procedure to follow after a needlestick, and those who were unfamiliar wit the procedure were also more likely to over estimate the ideal window for starting post exposure prophylaxis. 33\% of participants would only take PEP if the injury warranted it. These findings were significantly associated with education but not job category. Age also played a significant role in the perception of risk and compliance. Only $9 \%$ of staff reported a needlestick in the past 5 years but in a high prevalence HIV country these people are at high risk.

Conclusions The training of laboratory staff needs to be undertaken with focus on those with lower education levels to ensure understanding. This will allow all employees to have the best information around needlesticks and post exposure prophylaxis.

\section{EXPOSURE TO STATIC MAGNETIC FIELDS INCREASES RISK OF ACCIDENTS AMONG WORKERS FROM A MEDICAL IMAGING DEVICE PRODUCTION FACILITY}

SI M Bongers, Slottje, Portengen, Kromhout. IRAS, Utrecht University, Utrecht, Nederland

\subsection{6/oemed-2013-101717.174}

Acute neurocognitive and balance effects, and reported health complaints such as vertigo related to static magnetic fields (SMF) from magnetic resonance imaging (MRI) devices were the reason to study the effects of these exposures on the occurrence of accidents. In this first retrospective cohort study among workers from a facility producing medical imaging devices we studied the association between SMF and occurrence of accidents in general and during commuting.

Methods SMF exposure was assessed with a manufacturing facility specific historical job exposure matrix linked to company records on individual job histories, resulting in two SMF exposure measures: exposure in the year of a self-reported accident (recent exposure) and cumulative exposure up to the end of the year an accident was reported (career exposure). Data on occurrence of accidents was collected through an online questionnaire (1479 participants, participation rate 30\%). Data on injuries and physician-treated injuries due to accidents in the past 12 months were analysed with logistic regression. Discrete-time survival analysis was applied to historic data on the reported year of the first ever (near) traffic accident during commute to and from work.

Results High recent SMF exposure was found to be related to an increased risk of accidents leading to injury (Odds Ratio 4.39, 95\% Confidence Interval 1.20-16.06) with a stronger effect for injuries treated by a physician (OR 6.28, 95\%CI 1.7023.26). High recent SMF exposure was associated with increased risk (Hazard Rate 3.21, 95\% Confidence Interval 1.36-7.18) of (near) accidents during commute from home to work, but not from work. No increased risks were found with respect to career exposure after adjustment for recent exposure. 